\title{
Electron Interferometry at Crystal Surfaces
}

\section{Citation}

Becker, R. S., J. A. Golovchenko, and B. S. Swartzentruber. 1985. Electron Interferometry at Crystal Surfaces. Physical Review Letters 55, no. 9: 987-990. doi:10.1103/physrevlett.55.987.

\section{Published Version}

doi:10.1103/PhysRevLett.55.987

\section{Permanent link}

http://nrs.harvard.edu/urn-3:HUL.InstRepos:29407045

\section{Terms of Use}

This article was downloaded from Harvard University's DASH repository, and is made available under the terms and conditions applicable to Other Posted Material, as set forth at http:// nrs.harvard.edu/urn-3:HUL.InstRepos:dash.current.terms-of-use\#LAA

\section{Share Your Story}

The Harvard community has made this article openly available.

Please share how this access benefits you. Submit a story.

\section{Accessibility}




\title{
Electron Interferometry at Crystal Surfaces
}

\author{
R. S. Becker, J. A. Golovchenko, and B. S. Swartzentruber \\ AT\&T Bell Laboratories, Murray Hill, New Jersey 07974 \\ (Received 10 April 1985)
}

\begin{abstract}
Electron standing waves formed in the vacuum gap between the probe and the sample of the tunneling microscope are observed. The sensitivity of the standing-wave positions and frequencies to the surface potential are demonstrated. Further effects possibly due to Bragg backscattering from the surface atomic planes of the sample are discussed.
\end{abstract}

PACS numbers: $73.20 . \mathrm{Cw}, 73.40 . \mathrm{Gk}$

The ability experimentally to excite $x$-ray standing waves of angstrom wavelengths enables researchers to probe atomic positions interferometrically in bulk crystals as well as at interfaces. ${ }^{1,2}$ These studies rely on the interference between incident and Bragg-reflected $x$-ray beams to create the standing-wave field. More recently, standing-wave effects have been observed at surfaces in a total reflection geometry ${ }^{3}$ where very glancing angles are required for the incident $x$ rays. It might be thought that it would be more convenient to use an electron beam for these purposes because of the comparable wavelength and the availability of high-brightness sources. On the other hand, the strongly interacting nature of low-energy electrons compared with kiloelectronvolt $\mathrm{x}$ rays causes complications such as multiple Bragg reflections and strong inelastic scattering which can seriously complicate the analysis of any application to which these standing waves might be applied. In the following we show that electron standing waves can be excited at crystal surfaces and under suitable conditions their properties analyzed in a straightforward manner. We will discuss the sensitivity of the analysis to various properties of the surface and, finally, comment on applications.

Historically, the approach we follow may be traced back to the work of Young, Ward, and Scire, ${ }^{4}$ who introduced a new device for probing surfaces. In its simplest form the device consists of a fine metal probe tip which is positioned piezoelectrically close enough to a sample surface so that when a modest voltage is applied across the probe-tip-sample gap, electrons may tunnel across. A feedback circuit adjusts the probetip-sample distance so that a constant predetermined tunnel current flows, and hence a fixed gap distance is maintained. The tip is then raster scanned laterally across the surface, and the feedback correction signal recorded as probe tip height varies to follow the signal, generating a topographic image. This device, at the time called the "topografiner" by its inventors, was refined more than ten years later in dramatic fashion by Binnig and Rohrer, who aptly renamed it the scanning tunneling microscope and demonstrated that it was capable of obtaining real-space images with atomic resolution on metal ${ }^{5-7}$ and semiconductor ${ }^{8}$ surfaces. We have constructed a tunneling microscope at AT\&T
Bell Laboratories and used it to excite and observe electron standing waves between the probe tip and a metal sample.

Such standing waves, which occur when the gap bias voltage exceeds the work function, are manifest as oscillations in the field emissions current as bias and or gap distance are varied. These oscillations, caused by interference between incident and reflected electrons in the positive kinetic-energy region of the vacuum gap (delineated by the classical turning point and the sample surface) were predicted theoretically by Gundlach $^{9}$ and appear to have been observed in some semiconductor devices consisting of metal-insulator semiconductor junctions ${ }^{10}$ and Ga-Al-As heterostructures. $^{11}$ Such experiments are limited by the breakdown fields of the dielectric insulator, limited dynamic range due to nonadjustable gap spacing, and loss of coherence due to inhomogeneities in gap spacing. Preliminary observations of oscillatory behavior in vacuum gap tunneling have also been reported by Binnig and Rohrer. ${ }^{12}$ We present here a comprehensive picture, and a test of the physical mechanism involved. Sensitivities to various atomic parameters are indicated, as well as practical implications of the phenomena for future measurements. Finally, we comment on the connection between our observations and the recent work on image surface states.

A schematic layout of the experiment and an energy diagram of the tip-gap-sample region is shown in Fig. 1. The experiment was performed in a UHV chamber at $2 \times 10^{-10}$ Torr with a gold (110)-oriented crystal sample that was argon-ion. sputtered and annealed at $600^{\circ} \mathrm{C}$. The probe tip was a $\mathrm{KOH}$-etched single-crystal (110) tungsten tip cleaned in situ by drawing $200-\mu \mathrm{A}$ field emission current for $20 \mathrm{~min}$. A careful search of the gold surface using the instrument in the tunneling microscope mode provided a region of the sample surface flat over $6 \times 10^{4} \AA^{2}$. This is the region where the standing-wave experiments were performed.

The energy diagram in Fig. 1 depicts electrons tunneling from tungsten tip to gold sample and shows the position of the Fermi levels, inner potentials, and vacuum-gap tunneling barrier including image potential and first-image correction. The logarithm of the electron probability density is shown for conditions of 


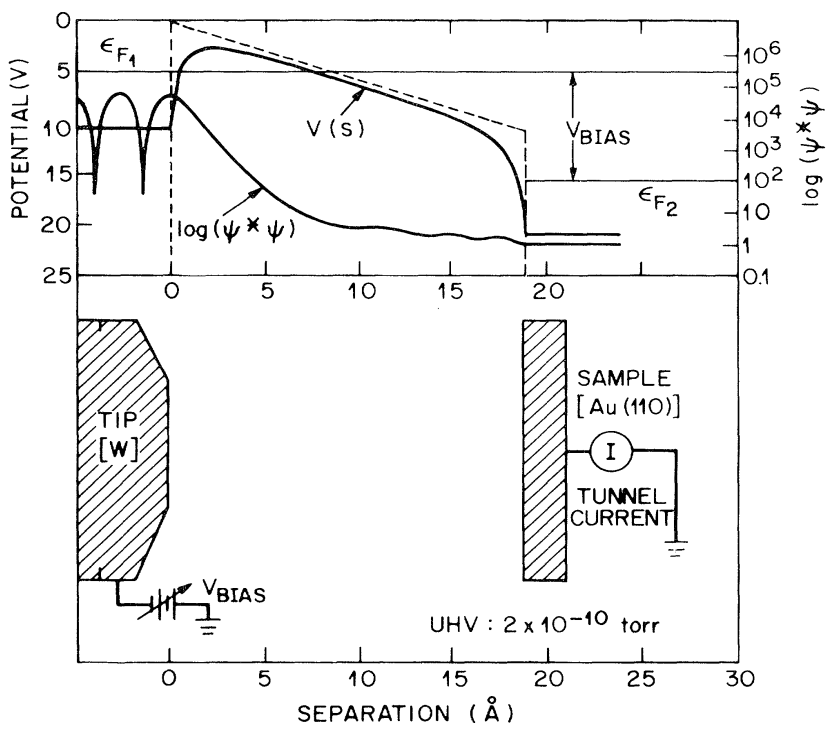

FIG. 1. Schematic illustration of experiment geometry and potential energy diagram used in calculations. The probability density for a normal electron at the tungsten Fermi surface is also shown.

gap spacing and bias corresponding to three standingwave antinodes in the gap. We obtain this result by numerical integration of the Schrödinger equation for the conditions shown.

To understand our experiment it is essential to realize that the tunneling microscope feedback mechanism is active during data acquisition. The feedback system is a dc integrating type, i.e., the loop gain rolls off at high frequency. The bias voltage has two components. A low-frequency ramp that scans from 1 to $20 \mathrm{~V}$ with the feedback system adjusts the gap distance to keep the tunnel current accurately at $1 \mathrm{nA}$ for this bias component. In addition there is a few hundred millivolt $6.4-\mathrm{kHz}$ modulation added to the gap bias which is too high in frequency for the feedback to respond. The current therefore contains a $6.4-\mathrm{kHz}$ component whose amplitude is recorded with a lockin amplifier and is referred to in the discussion below as $d I / d V$. Also recorded is the position signal which adjusts the gap as the bias voltage ramps in order to maintain an average tunnel current of $1 \mathrm{nA}$.

The results are shown in Fig. 2. Eleven oscillations in $d I / d V$ are clearly seen extending from about 5 to $18 \mathrm{~V}$ while the tip retreats nearly $30 \AA$. It is interesting to note that it would be extremely difficult to obtain the large number of oscillations shown if the experiment had been performed by varying only the bias voltage or gap distance, since then the dynamic range required of the current measurement would be unattainable for the same number of oscillations. Also shown in Fig. 2 is a plot of gap distance. Since we ac-

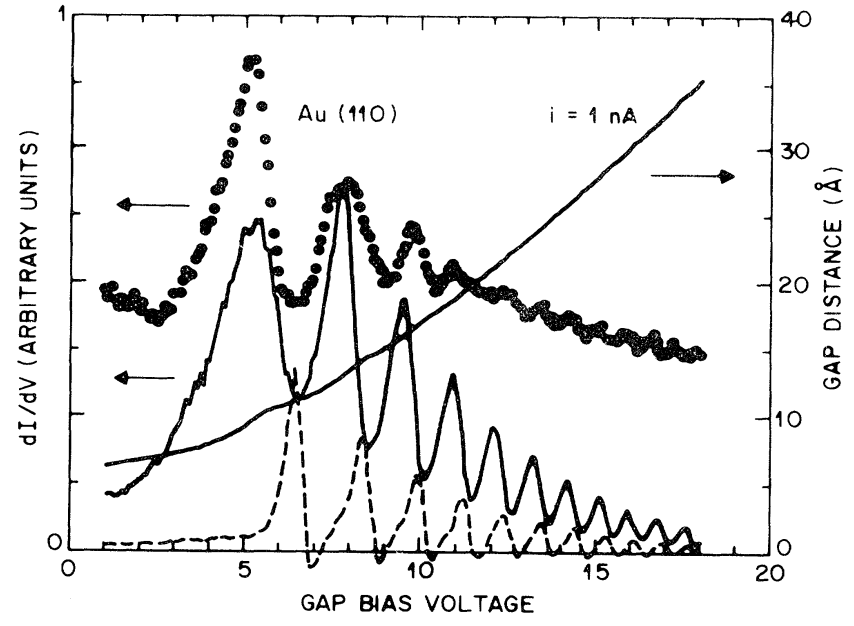

FIG. 2. Experimental curves of $d I / d V$ (closed circles) and gap distance vs bias voltage. The oscillatory solid curve is theoretical barrier penetration factor. The dashed curve omits the image-potential contribution.

tually only have a measure of change in tip position it is not possible for us to place the gap-distance origin accurately by only experimental means. In order to obtain the offset the one-dimensional Schrödinger equation is integrated to yield the transmission probability for the observed relative path as a function of offset. Only for the offset shown is a constant probability obtained corresponding to the fixed tunnel current flowing as the bias is scanned.

The oscillatory solid curve in Fig. 2 is again obtained from an integration of the Schrödinger equation along the gap-bias path just described. The agreement between peak and valley positions is quite good, although the modulation observed experimentally is certainly smaller than that calculated. By comparing absolute predictions of tunneling current per unit area with our data we also estimate the effective tunneling area to be $\sim 200 \AA^{2}$. As a final check on the consistency of these calculations the results of Simmons ${ }^{13}$ were used in the Fowler-Nordheim tunneling region and good agreement was also obtained for the gap-bias path as expected. The oscillatory behavior is not obtained in such a calculation where the WKB approximation has been made and reflected waves have been neglected.

Figure 2 illustrates the sensitivity of the standing waves to the shape and magnitude of the potential near the sample surface. The dashed curve is a theoretical calculation neglecting the effect of image potential. In order to compensate for the increased barrier height, the tungsten work function had to be lowered $0.5 \mathrm{~V}$ to reach the same tunneling probability. It is immediately apparent that the frequency of the 
oscillations has shifted slightly, along with the apparent beginning of the oscillation region. More striking is the large increase in finesse due to the sharper barrier transition causing increased reflectivity. This is certainly not in the direction of obtaining better agreement with data. Calculations averaging over incident electron direction out to $20^{\circ}$ in a $10-\mathrm{mV}$ region around the tungsten Fermi level tend to wash out the negative resistance regions of the new curve but do not alter the frequency or overall finesse of this electron-wave Fabry-Perot device.

Trial and error shows that the fit of the theory and data is far more dependent on the selection of the gap-distance origin than on the work functions of either the tip or sample. Errors of $1 \AA$ will produce tunneling currents that vary by two decades or more. Small variations of the work function of the tip tend to shift the vertical position of the whole curve. Finally, as expected, variation of the sample work function directly influences the apparent origin of the standingwave oscillations in the $d I / d V$ curves.

Close examination of the data in Fig. 2 reveals a feature in the data yet to be discussed. Between the fourth and fifth oscillation in the $d I / d V$ data, a disruption appears in the decaying periodic structure of the curve and the relative oscillation amplitude may actually increase somewhat at higher bias. This effect has been repeatedly observed in other data runs as well. We suggest that the origin of this effect is connected with the crystalline nature of the sample. Back reflections may not only be associated with the sample surface but also with atomic planes below the surface. Perusal of electron mean free paths in gold ${ }^{14}$ suggests that the electron wave function maintains coherence over distances of order $15 \AA$ before inelastic scattering depletes the state. This suggests that a few atomic planes may be accessible and their effect should be included in the calculation of $d I / d V$. This is beyond the scope of our present purpose and we leave this aspect of our results simply by noting that empirically, at least in the case we have studied, the surface reflection seems to be the dominant channel.

Figure 3 illustrates, in more detail, the standingwave nature of the probability density in the kineticenergy region of the gap. Considered here are normal propagating electrons from the tip Fermi level to the sample for several points on the curve of feedbackstabilized gap distances vs bias voltage. Note how each successive oscillation in $d I / d V$ incorporates an additional standing wave in the gap. Note also that the standing-wave interference effects can hardly be associated with strongly resonant energy levels (long lifetimes) in the vacuum gap since the electrons spend hardly any excess time there as a result of the relatively small reflection coefficient at the sample surface.

At this point we comment briefly on the question of

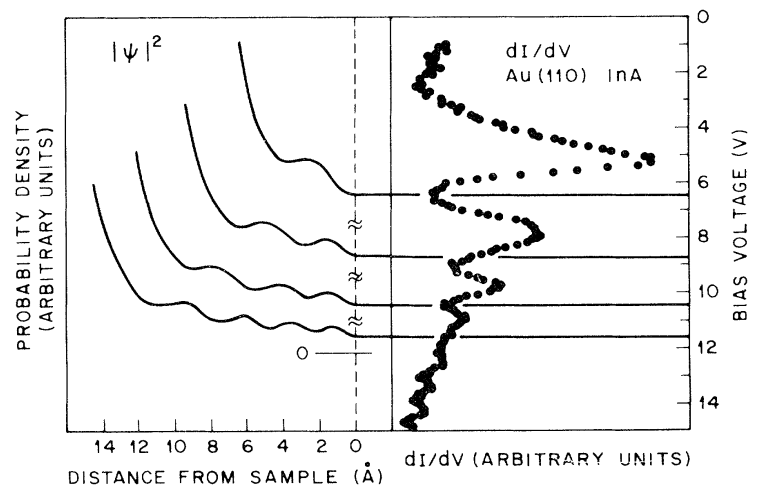

FIG. 3. Electron probability density in the vacuum gap for several operating points on the $d I / d V$ curve. The probability curves are shifted vertically so as to intersect the appropriate operating point on the right.

the role of image-potential states in explaining our observations. Cole and Cohen ${ }^{15}$ originally called attention to the existence of such states which are found in band gaps near the vacuum level. Image-potential states are of recent interest because they appear to be experimentally observed in inverse photoemission ${ }^{16,17}$ on metals. Garcia ${ }^{18}$ has recently suggested that resonant tunneling into such states can be responsible for much of the low-field structure in tunneling measurements of the type presented here. While this proposal may apply to certain special cases where band gaps may be present near the vacuum level, it has little bearing on our experiment where no such band gaps exist between the vacuum level and the highest-energy data we have taken. ${ }^{19}$ Even under conditions where such states may exist, by their very nature they are excluded from the bulk and therefore cannot contribute to a net current normal to the surface.

We conclude this Letter by noting that we have observed extended electron standing-wave effects in the vacuum tunneling transmission probability between tungsten and gold surfaces. A quantitative understanding of the effect requires detailed knowledge of the tip-vacuum-sample junction including potentials and distances. The latter makes the effect particularly useful in calibrating tunneling microscope tip positions on the subangstrom scale. When the role of crystal reflections and inelastic effects are sorted out we believe that valuable surface potential information will be available. We stress that the actual areas probed in such a measurement are quite small (though not of atomic dimensions) and variation of the parameters discussed above can serve as the source of contrast for the microscopy of practical structures on sub-1000- $\AA$ scales. Finally, we envision utilizing the standing waves observed in the experiments in an analogous way to that reported in Refs. 1-3 to probe (perhaps 
through an inelastic channel) details of atomic positions normal to the surface and measured relative to the bulk crystal below the surface.

1J. A. Golovchenko, J. R. Patel, D. R. Kaplan, P. L. Cowan, and M. L. Bedzyk, Phys. Rev. Lett. 49, 560 (1982).

2J. R. Patel, J. A. Golovchenko, R. J. Morris, and J. C. Bean, Phys. Rev. B 31, 6884 (1985).

${ }^{3}$ R. S. Becker, J. A. Golovchenko, and J. R. Patel, Phys. Rev. Lett. 50, 153 (1983).

${ }^{4}$ R. Young, J. Ward, and F. Scire, Phys. Rev. Lett. 27, 922 (1971), and Rev. Sci. Instrum. 43, 999 (1972).

${ }^{5}$ G. Binnig, H. Rohrer, Ch. Gerber, and E. Weibel, Surf, Sci. 126, 236 (1983).

${ }^{6} \mathrm{G}$. Binnig, H. Rohrer, Ch. Gerber, and E. Weibel, Surf. Sci. 131, L379 (1983).

${ }^{7}$ A. M. Baro, G. Binnig, H. Rohrer, Ch. Gerber, H. Stoll, A. Baratoff, and F. Salvan, Phys. Rev. Lett. 52, 1304
(1984).

${ }^{8}$ G. Binnig, H. Rohrer, Ch. Gerber, and E. Weibel, Phys. Rev. Lett. 50, 120 (1983).

${ }^{9}$ K. H. Gundlach, Solid State Electron. 9, 949 (1966).

10 J. Maserjian and N. Zamani, J. Appl. Phys. 53, 559 (1982).

${ }^{11}$ T. W. Hickmott, P. M. Solomon, R. Fischer, and H. Morkoc, Appl. Phys. Lett. 44, 90 (1984).

${ }^{12} \mathrm{G}$. Binnig and H. Rohrer, Helv. Phys. Acta 55, 726 (1982).

13J. G. Simmons, J. Appl. Phys. 34, 1793 (1963).

${ }^{14}$ M. P. Seah and W. A. Dench, Surf. Interface Anal. 1, 1 (1979).

${ }^{15}$ M. W. Cole and M. H. Cohen, Phys. Rev. Lett. 23, 1238 (1969).

${ }^{16}$ V. Dose, W. Altmann, A. Goldman, U. Kolac, and J. Rogozik, Phys. Rev. Lett. 52, 1919 (1984).

${ }^{17}$ D. Straub and F. J. Himpsel, Phys. Rev. Lett. 52, 1922 (1984).

${ }^{18}$ N. Garcia, Bull. Am. Phys. Soc. 30, 251 (1985).

${ }^{19}$ H. Eckardt, L. Fritsche, and J. Noffke, J. Phys. F 14, 97 (1984). 